\title{
Wood Classification Based on Edge Detections and Texture Features Selection
}

\author{
Achmad Fahrurozi $^{1}$, Sarifuddin Madenda ${ }^{1}$, Ernastuti $^{1}$, Djati Kerami ${ }^{2}$ \\ ${ }^{1}$ Computer Science Department, Gunadarma University, Indonesia \\ ${ }^{2}$ Departement of Mathematics, University of Indonesia, Indonesia
}

\section{Article Info \\ Article history: \\ Received Feb 21, 2016 \\ Revised Jul 19, 2016 \\ Accepted Jul 30, 2016}

\section{Keyword:}

Edge detection

GLCM

Naïve-bayes classifier

Texture features

Wood quality classification

\begin{abstract}
One of the properties of wood is a mechanical property, includes: hardness, strength, cleavage resistance, etc. Among these properties there that can be measured or estimated by visual observation on cross-sectional areas of wood, which is based on inter-fiber density, fiber size, and lines that build the annual rings. In this paper, we proposed a new wood quality classification method based on edge detections. Edge detection is applied to the wood test images with the aim to improving the characteristics of wood fibers so as to make it easier to distinguish their quality. Gray Level Cooccurrence Matrix (GLCM) used to obtain wood texture features, while the wood quality classification done by Naïve Bayes classifier. Found in our experimental results that the first-order edge detection is likely to provide a good accuracy rate and precision. The second order edge detection is highly dependent on the choice of parameters and tends to give worse classification results, as filtering the original wood image, thus blurring characteristics related to wood density. Selection of features obtained from co-occurrence matrix is also quite affected the classification results.
\end{abstract}

Copyright (C) 2016Institute of Advanced Engineering and Science. All rights reserved.

\section{Corresponding Author:}

Achmad Fahrurozi,

Computer Science Department,

Gunadarma University,

100 Margonda Road, Depok, West Java, Indonesia.

Email: achmad.fahrurozi12@gmail.com

\section{INTRODUCTION}

The determination of wood quality is especially important for a manufacturer of wood, as a means of quality control which serves to determine the appropriate price for each wood product and maintain consumers' confidence. The quality of wood is naturally influenced by several factors, including the density of the wood structure fiber, shelter, weather and so forth. Therefore, wood will have several of mechanical properties, textures, shapes and colors. The determination of wood quality can be based on several things, including slope of the wood fiber [1], defects in the wood, especially knots [2] or the mechanical properties of wood [3],[4]. Hardness and stiffness are mechanical properties that frequently observed and considered to determine quality of wood [4], and have positive correlation to density of wood [5]. So the alleged of observations are based on the density between constituent of annual rings. The number of lines that build annual rings of four-seasons wood usually more than the wood in two-seasons region or tropical. The distance the lines on annual rings determined by large-small and density the constituent fibers of the wood.

At the simplest level, the assessment of quality of wood carried through visual observation by experts who are experienced [6]. But it is tend to be less efficient in terms of time and effort. Over the time, the assessment of the quality of wood can also be done in the laboratory, where testing can be either destructive or nondestructive [3]. As the development of current technology, there are lot of research has been done to replace the function of the human eye as a tool of visual observations, one of whom is use digital 
cameras. By using a digital camera we can obtain a digital image of an object. Digital image obtained can be read and processed by a computer, to subsequently use in the necessary research. Digital image-based research has been developed for considering the characteristics of the object in a digital image that contains the object. Nondestructive methods related to wood quality tests have been done such as by using a wood image based on the slope of the wood fiber [1] or considering the number of defects and their distributions [7]. This study focuses on the classification of wood quality using a wood image, but is observed a test wood texture by using texture features of wood. Texture features obtained by using Gray-Level Co-occurrence Matrix (GLCM) as an extractor which is implemented in Matlab software. Texture features of GLCM that often used in research of texture analysis are Contrast, Correlation, Inverse Difference Moment (IDM), Entropy, Energy and Homegenity [8]-[13]. Before feature extraction, edge detection carried, because the characteristics of the edges in an image can be used to represent the image to further analysis and implementation [14], and also reinforces the observation of fiber density and annual rings on the of wood were observed in this study.

\section{THEORITICAL CONCEPTS}

\subsection{Edge detections}

Edge detection is a method used to gain an edge contained in an image observed. Edge states limit where there has drastic change of gray level [15]. In general, edge detection method is divided into two, ie first-order edge detection methods (includes Roberts, Prewitt and Sobel) and second-order edge detection method (Canny and Laplacian of Gaussian filters). Roberts detector using a 2x2 mask or kernel, where the differential sought by diagonally (45 and 135 degrees) as illustrated in the Figure 1.
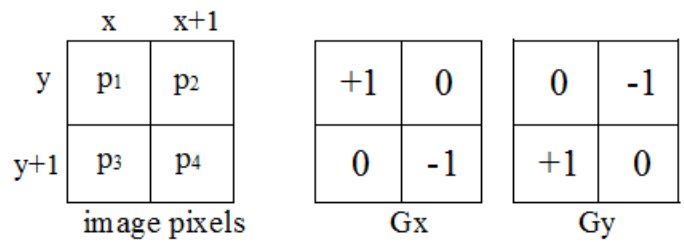

Figure 1. Relationship between image pixels and mask of Roberts operator

Thus, Roberts operator detects the edges in a diagonal direction. The pixel intensity values in matrices as Roberts operator results at position $(x, y)$ on the this paper is calculated by the formula:

$$
\mathrm{r}(x, y)=\left|\mathrm{p}_{1}-\mathrm{p}_{4}\right|+\left|\mathrm{p}_{3}-\mathrm{p}_{2}\right|
$$

Meanwhile, Prewitt and Sobel are edge operators that use a 3x3 kernel as shown in the Figure 2.

\begin{tabular}{|c|c|c|}
\hline+1 & 0 & -1 \\
\hline+1 & 0 & -1 \\
\hline+1 & 0 & -1 \\
\hline \multicolumn{4}{|c|}{$\mathrm{Gx}$}
\end{tabular}

(a)

\begin{tabular}{|c|c|c|}
\hline-1 & -1 & -1 \\
\hline 0 & 0 & 0 \\
\hline+1 & +1 & +1 \\
\hline \multicolumn{3}{|c|}{ Gy }
\end{tabular}

\begin{tabular}{|c|c|c|}
\hline-1 & 0 & +1 \\
\hline-2 & 0 & +2 \\
\hline-1 & 0 & +1 \\
\hline \multicolumn{3}{|c|}{$\mathrm{Gx}$}
\end{tabular}

(b)

\begin{tabular}{|c|c|c|}
\hline+1 & +2 & +1 \\
\hline 0 & 0 & 0 \\
\hline-1 & -2 & -1 \\
\hline \multicolumn{3}{|c|}{ Gy }
\end{tabular}

Figure 2. Kernel of (a) Prewitt operator and (b) Sobel operators

From Figure 2, we can see that both Prewitt and Sobel operator detects the edges in vertical and horizontal directions. Canny edge detection perform smoothing in advance using Gaussian function and is claimed as the best edge detection method because it can capture both edges of the strong and weak edges, but has complex computation and time consuming [14],[16]. Laplacian of Gaussian (LoG) has a kernel size is varied and requires $\sigma$ (sigma) as a parameter, where sigma is parameter used in LoG's formula [14]: 


$$
\operatorname{LoG}(x, y)=\frac{1}{\pi \sigma^{4}}\left[1-\frac{x^{2}+y^{2}}{2 \sigma^{2}}\right] e^{\frac{-\left(x^{2}+y^{2}\right)}{2 \sigma^{2}}}
$$

Generally, the relationship between value of sigma and the size of the kernel that is often used is size $=$ ceiling $(3 *$ sigma $) * 2+1$.

\subsection{Gray-level co-occurrence matrix (GLCM)}

Gray-Level Co-occurrence Matrix (GLCM) is a method often used in the texture analysis [17], proposed by Haralick in [18] on 1973 that includes 14 features texture. GLCM produces features which describe well the relationship of adjacency among pixels in an texture image. These features extracted from a co-occurrence matrix, where the construction of these matrices depends on several parameters, including: spatial distance $(d)$, direction or angle orientation $(\theta)$ and gray-limits $(G)$. Spatial distance determines adjacency distance among pixels of an image, while the direction determines how to neighboring pixels on the specified direction. Gray-limits parameter determines the size of the co-occurrence matrix are generated, as well partitioned intensity value contained in the entire image to produce a matrix that is "converted". Figure 3 shows an example of the relationship between co-occurrence matrix construction and the selected parameter values.

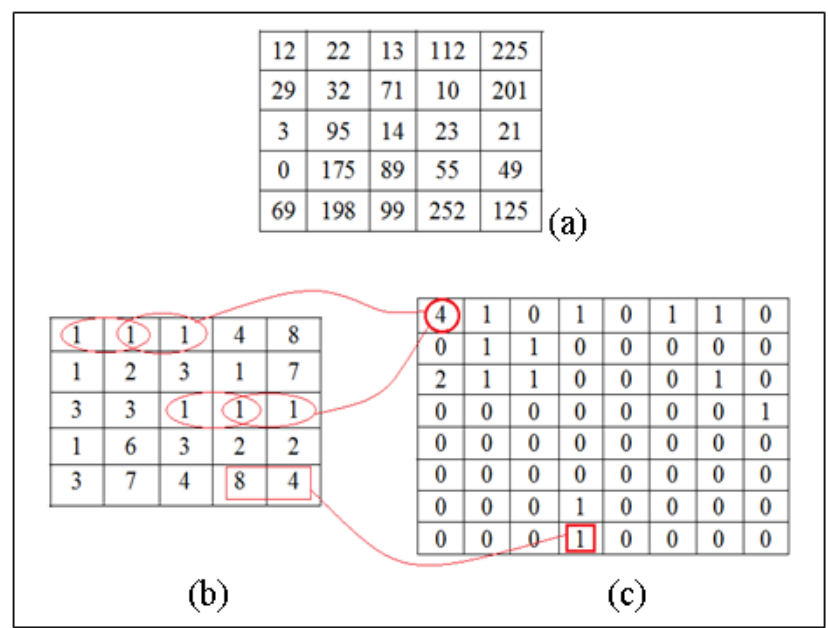

Figure 3. Illustration of GLCM constructions: (a) matrix of image I, (b) converted matrix, (c) co-occurrence matrix obtained by choosing $d=1, \theta=0$ and $G=8$

As figure above, Figure 3-a is a matrix representation of an image I with the assumption have gray level $0-255$, while Figure3-b describes converted matrix by $G=8$, with rules: intensity from $h^{*} 2^{5}$ to $(h+1)^{*}\left(2^{5}-1\right)$ changed to $(h+1)$, where $h=0,1,2, \ldots, 6,7$.

\subsection{Naïve-Bayes classifier}

Naïve-Bayes classifier works using the principle of Naïve-Bayes, the conditional probability to events mutually independent. For example given the task of classification which consists of two classes, where each class is recognized or determined by considering three features, the Naïve-Bayes classifier determines an event or item into the particular class by first calculating the probability that an item goes into each a given class, in this suppose that $\mathrm{c}_{1}$ and $\mathrm{c}_{2}$ are class, then the probabilities denoted by $\operatorname{Pr}\left(\mathrm{c}_{1} \mid \mathrm{x}\right)$ and $\operatorname{Pr}$ $\left(c_{2} \mid x\right)$. Suppose item $x$ has the value of a particular feature, ie $f_{1}, f_{2}$ and $f_{3}$. Then the calculation is based on the assumption of independence of each feature of an item $\mathrm{x}, \operatorname{Pr}\left(\mathrm{c}_{\mathrm{i}} \mid \mathrm{x}\right)$ is calculated by the formula:

$$
\begin{aligned}
& \operatorname{Pr}\left(\mathrm{c}_{1} \mid \mathrm{x}\right)=\operatorname{Pr}\left(\mathrm{c}_{1} \mid \mathrm{f}_{1}\right)^{*} \operatorname{Pr}\left(\mathrm{c}_{2} \mid \mathrm{f}_{2}\right)^{*} \operatorname{Pr}\left(\mathrm{c}_{1} \mid \mathrm{f}_{3}\right) \text { and } \\
& \operatorname{Pr}\left(\mathrm{c}_{2} \mid \mathrm{x}\right)=\operatorname{Pr}\left(\mathrm{c}_{2} \mid \mathrm{f}_{1}\right)^{*} \operatorname{Pr}\left(\mathrm{c}_{2} \mid \mathrm{f}_{2}\right)^{*} \operatorname{Pr}\left(\mathrm{c}_{2} \mid \mathrm{f}_{3}\right)
\end{aligned}
$$

If $\operatorname{Pr}\left(c_{1} \mid x\right)>\operatorname{Pr}\left(c_{2} \mid x\right)$ then the item $x$ would be classified as a member of the class $c_{1}$ and vice versa. If there are more than two classes, then the item will be placed into a class with the greatest probability. Naïve Bayes classifier has two primary outputs, ie posterior matrix and vector prediction. Matrix contains the 
probability that some items to be classified to belong to each class, and the prediction vector is a vector that expressed the class chosen for that items.

\section{PROPOSED METHOD}

In this study the classification of wood based on its quality by first applying edge detection on the original wood image. Edge detection operators used in our proposed method are Roberts, Sobel, Prewitt, Canny and LoG (with some parameter value). Extraction of texture features performed using GLCM and Naïve Bayes is adopted for classifying the different qualities of wood. The proposed method is summarized on the diagram in Figure 4 below:

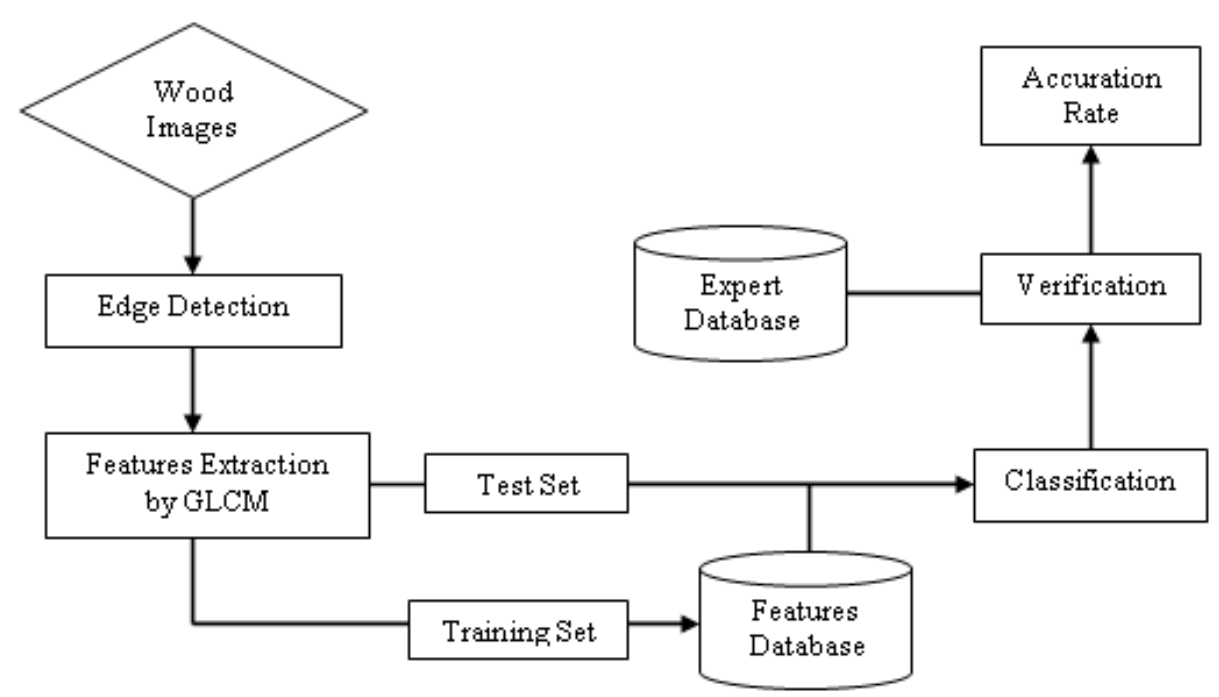

Figure 4. Scheme of the proposed method

In this method, we use four textural features, (i.e. Contrast, Correlation, Energy and Homogeneity) derived from the co-occurrence matrix with various values of GLCM parameters. We have four kinds of wood quality (as expert database, see Figure 4), where each type is composed of 20 images, which are divided into two sets (i.e. test set and training set, each containing 10 images). GLCM texture features from all images in the training set are stored as a feature database, that is then used to perform the classification of the images in the test set. The wood quality classification results obtained by Naive Bayes classifier will be compared or verified by the expert classification results that are in the expert database. And as final output of the proposed classification method is the accuration rate, with precision are optional outputs. Accuration rate compute by dividing the number of all correct predicted items in test set with the number of items in test set. Precision compute depend on the query (in our paper, the possible query is label of wood type, i.e. TF, F, M and GG).

\section{WOOD IMAGE}

This section will be divided into two parts. The first part shows different kinds of wood quality based on visual texture features present in wood images that will be tested. The second part discusses about the results of various edge detection methods on wood image.

\subsection{Wood image acquisition}

As the results of wood image acquisition, we have four types of wood which are distinguished by their quality. These images are provided by LE2I laboratory, Université de Bourgogne, France. There are 80 images from 80 samples of wood, which are grouped into four types of quality and each group consists of 20 samples. Figure 5 shows an example of wood images with four different qualities. These images are grayscale and in png format, wherein appears the transverse cross-sections, which reveal the annual rings and the rays of wood. 


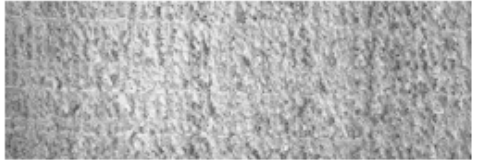

(a)

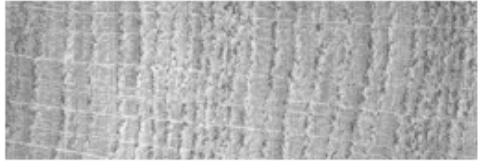

(c)

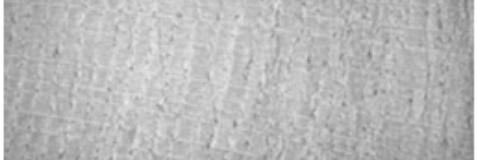

(b)

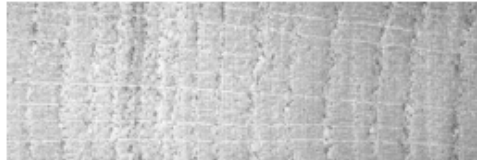

(d)

Figure 5. Image of four qualities of wood: (a) very good, (b) good, (c) medium and (d) poor

\subsection{Edge detection images}

The purpose of the edge detection applied to the wood image is to further strengthen the appearances of the wood texture features contained therein, especially to obtain the fiber composition and the lines that construct annual rings that are considered as parameters to determine the quality of wood. Figure 6 shows edge detection results of several methods (i.e. Roberts, Sobel, Prewitt, Canny and LoG) applied to original wood image in Figure 5-b.

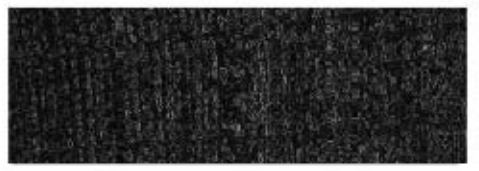

(a)

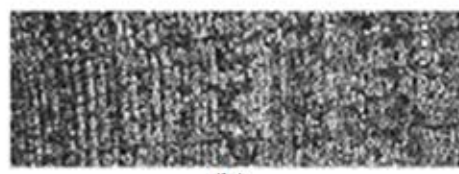

(b)

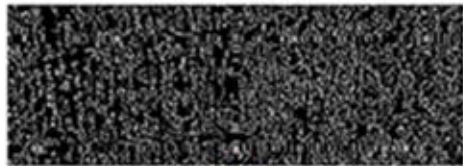

(d)

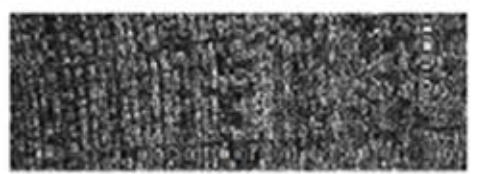

(c)

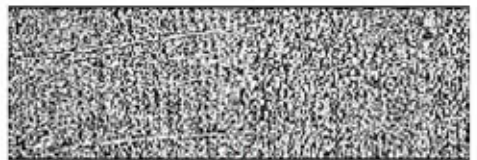

(e)

Figure 6. Edge detection results of several operators: (a) Roberts, (b) Sobel, (c) Prewitt, (d) Canny and (e) LoG (with parameter $\sigma=0.66$ and size 5)

We can see in Figure 6 that the results of Sobel and Prewitt operators have similar characteristics as mentioned in [14]. Image of second order edge detection methods have characteristics much different than the results of the first order. Canny detector generates the edges in binary format, that can capture the strong and weak edges, but these edges are very thin compared to the others operators results. The output of LOG operator seems like the original image with reinforcement on the view of fibers.

\section{E XPERIMENTAL RESULT}

This section presents the analysis of GLCM texture features, effect of change GLCM parameters, and classification results using our proposed method. As explained in the first part, that this study was conducted to classify wood based on its quality with a focus on utilizing the image of the test wood. Because previous research related to the mechanical properties of wood is done by using ultrasonic waves, $\mathrm{x}$-rays and other laboratory equipment (not use image of wood), then the comparison against the results in this paper 
aren't done. Research on the image of wood is generally performed to detect wood defects [1],[7] or to classify wood based on species [9]-[13]. Therefore, we present experimental results of classification of wood database that we use in this paper were implemented in some classification method based on wood species.

\subsection{Wood Classification of Proposed Method}

As explained earlier that GLCM as features extractor has three parameters, i.e. spatial distance, direction and gray limits. The selection of different values of these parameters can affect the results of statistical that calculate from co-occurence matricex, that maybe influence classification results. Some edge detection methods also consider the orientation towards the edges, so in this paper, change of these three parameters are considered for texture features extraction that use as classification features. Table 1 presents the differences of classification result (as measured by accuration rate, precision, and running time) that distinguished by the use of edge detection methods and the selection of spatial distance. Accuration rate and precision are presented in percent, and running time in second. In this paper, we present the average of precision of all possible queries (i.e. TF, F, M and GG).

Table 1. Classification Results vs Spatial Distance Value Based on Various Edge Detection Methods

\begin{tabular}{ccccccc}
\hline \multirow{2}{*}{ Operator } & Evaluation & \multicolumn{5}{c}{ Spatial Distance } \\
& Accuration Rate & 1 & 2 & 3 & 4 & 5 \\
\hline \multirow{2}{*}{ Roberts } & Precision & 87.4126 & 88.3117 & 88.3117 & 86.6071 & 88.3117 \\
& Running Time & 13.6997 & 13.7156 & 13.7080 & 13.8462 & 13.6953 \\
& Accuration Rate & 82.5 & 87.5 & 82.5 & 85 & 82.5 \\
Sobel & Precision & 86.4177 & 89.3939 & 86.4177 & 88.6905 & 87.0879 \\
& Running Time & 44.8902 & 44.9442 & 44.8890 & 44.8170 & 44.8790 \\
& Accuration Rate & 82.5 & 87.5 & 82.5 & 85 & 85 \\
Prewitt & Precision & 86.4177 & 89.3939 & 86.4177 & 88.6905 & 88.6905 \\
& Running Time & 44.8971 & 44.8067 & 44.8900 & 44.8422 & 44.8321 \\
& Accuration Rate & 80 & 75 & 52.5 & 52.5 & 47.5 \\
Canny & Precision & 80.5240 & 77.6380 & 50.0000 & 54.1558 & 46.9697 \\
& Running Time & 23.5565 & 21.9989 & 22.1658 & 22.1820 & 22.0603 \\
& Accuration Rate & 77.5 & 82.5 & 77.5 & 80 & 80 \\
& Precision & 77.4784 & 83.0186 & 77.0617 & 81.0956 & 82.3593 \\
& Running Time & 18.2749 & 18.2295 & 18.1644 & 18.1959 & 18.1093 \\
\hline
\end{tabular}

In Table 1 it can be seen that changes in the value of spatial distance parameter doesn't give a significant effect on the classification results, which is characterized by a constant percentage of accuration rate and precision, except for Canny operator. While the impact of changes to the $G$ against classification results are presented in Table 2 .

Table 2. Classification Result vs Gray Limits Value Based on Various Edge Detection Methods

\begin{tabular}{|c|c|c|c|c|c|c|}
\hline \multirow{2}{*}{ Operator } & \multirow{2}{*}{ Hal yang Diamati } & \multicolumn{5}{|c|}{ Gray limits } \\
\hline & & 8 & 16 & 32 & 64 & 128 \\
\hline \multirow{3}{*}{ Roberts } & Accuration Rate & 85 & 82.5 & 82.5 & 82.5 & 82.5 \\
\hline & Precision & 87.4126 & 86.0390 & 86.4177 & 86.4177 & 86.4177 \\
\hline & Running Time & 13.6997 & 14.7736 & 14.9804 & 15.0280 & 15.4367 \\
\hline \multirow{3}{*}{ Sobel } & Accuration Rate & 82.5 & 82.5 & 82.5 & 85 & 80 \\
\hline & Precision & 86.4177 & 86.4177 & 86.4177 & 87.7914 & 79.7115 \\
\hline & Running Time & 44.8902 & 45.9572 & 45.9146 & 45.8720 & 46.283 \\
\hline \multirow{3}{*}{ Prewitt } & Accuration Rate & 82.5 & 82.5 & 82.5 & 82.5 & 82.5 \\
\hline & Precision & 86.4177 & 86.4177 & 86.4177 & 86.4177 & 86.4177 \\
\hline & Running Time & 44.8971 & 45.8353 & 45.7818 & 45.8808 & 46.3403 \\
\hline \multirow{3}{*}{ LoG } & Accuration Rate & 77.5 & 77.5 & 77.5 & 80 & 80 \\
\hline & Precision & 77.4783 & 77.4784 & 77.4784 & 80.8333 & 80.8333 \\
\hline & Running Time & 18.2749 & 18.3768 & 18.1974 & 18.3390 & 18.6414 \\
\hline
\end{tabular}

Based on the results obtained in Table 1 and Table 2, we can summarize that Roberts operator generally provides the best classification results, both in terms of accuration rate, precision, running time and the stability of level of accuracy. Change the parameters of spatial distance and graylimits not provide a considerable influence on the accuration rate and precision, except on Canny operator. Thus, the best value for a parameter $\mathrm{G}$ that can be selected is 8 , as mentioned in [19] with the intention of reducing the computation, because the size of co-occurrence matrix is smaller than the current $\mathrm{G}$ value greater than 8 . 
Further analysis carried out on the angle orientation parameter and Roberts operator, due only Roberts operator using a diagonal direction to the determination of the edge and give the best classification result constantly. Then research did by using the features GLCM with the parameters obtained from a diagonal direction, ie 45 and 135 degrees. Results of classification by using the image of Roberts operator shown in Table 3.

Table 3. The Effect of Changing Features Selection on Roberts Image

\begin{tabular}{ccc}
\hline \multirow{2}{*}{ Direction } & \multicolumn{2}{c}{ Spatial } \\
& 1 & 2 \\
\hline $0,45,90$ and 135 & 85 & 85 \\
45 and 135 & 87.5 & 85 \\
\hline
\end{tabular}

It can be seen from Table 3 that the classification results using the features from the direction 45 and 135 degrees with the spatial distance 1 provides a better accuration rate than using all the features that come from the four directions of GLCM on Roberts image.

\subsection{Wood Classification of Other Method}

Classification method used here is the classification of wood based on species, proposed by Mohan S. in [12]. This method uses Canny operator at pre-processing stage and uses three texture features of GLCM ie Contrast, Entropy, and Standard Deviation. The number of features used is 30, because it uses block image from the original image. Classifier is used Pearson correlation factor. To determine the class of an image in test set (hereinafter referred to test sample), calculated the correlation factor between the test sample with each image in training set. Suppose the highest correlation factor is with an image included in the class $\mathrm{K}$, then test sample classified as wood of class K. This method looks like k-NN classifier with correlation distance and $k=1$. The database and the test set described on third part in this paper implemented to this method then compared the results with our proposed method. Classification results of this method presented in Table 4, where items in the training set be numbered from 01 to 40 and items in the test set be numbered from 41 to 80 .

Table 4. Implementation Results of Data of Wood-Based Quality on Mohan's Classification Method

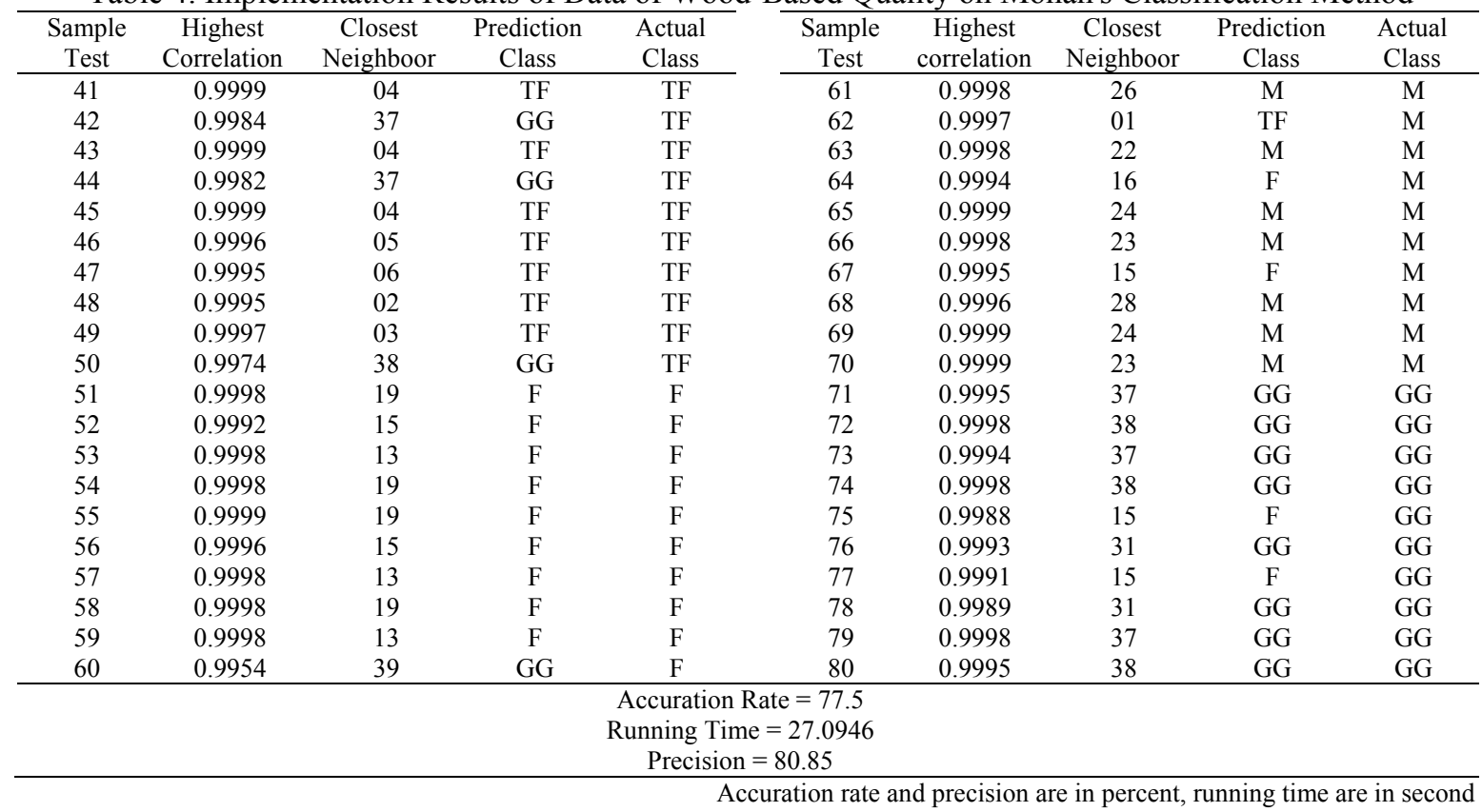

In Table 4 above, the closest sample in question is an object or image in the training set that has the highest correlation value against to test sample compared to other objects in the training set. It can be seen that the accuracy and precision of classification method by Mohan S is quite low, and no single type of wood that has a precision up to $100 \%$. 


\section{CONCLUSION}

Based on the classification results obtained, edge detection method of the first order gives the image that characterizes the spread of fiber which is good for each wood group. Canny are less suitable for use in the case of wood classification based on quality, because it captures the weak edge though, so obscure the characteristic that can distinguish among types of wood. Pre-processing using edge detection methods in our proposed method, especially the first order, give a fairly good classification results. Roberts operator is relatively more efficient because of the number of features used can be reduced to half and provide the better accuration rate on spatial distance of 1 , and generally its performance is comparable to others detector that use more features. Wood classification method based on species by Mohan S. less suitable if it is implemented to the data in this study that is wood-based quality. It is alleged the use of Canny operator and because the data characteristics of wood-based spasies not the same as the data characteristics of wood-based quality that used in this study. The selection of features through the determination of the parameters that construct GLCM also very important because quite affects the accuracy and precision from the classification process, especially that use Roberts operator in pre-processing stage.

\section{REFERENCES}

[1] R. W. Atmaja, et al., "The Detection of Straight and Slant Wood Fiber Through Slop Angle Fiber Feature," TELKOMNIKA Indonesian Journal of Electrical Engineering, vol/issue: 14(2), pp. 318-322, 2015.

[2] W. Lin and J. Wu, "Nondestructive Testing of Wood Defects Based on Stress Wave Technology," TELKOMNIKA Indonesian Journal of Electrical Engineering, vol/issue: 11(11), pp. 6802-6807, 2013.

[3] T. R. Mardikanto, et al., "Sifat Mekanis Kayu," PT Penerbit IPB Press, 2011.

[4] L. Karlinasari, "Non-destructive ultrasonic testing method for determining bending strength properties of Gmelina wood (Gmelina arborea)," Journal of Tropical Forest Science, vol. 20, pp. 99-104, 2008.

[5] J. Baan, et al, "Prediction of Mechanical Properties-Modulus of Rupture and Modulus of Elasticity-of Five Tropical Species by Nondestructive Methods," Maderas, Ciencia y Tecnologia, vol/issue: 17(2), pp. 239-252, 2015.

[6] V. Bucur, "Nondestructive Characterization and Imaging of Wood," Springer-Verlag Berlin Heidelberg, 2003.

[7] H. I. A. Wibowo and F. Meriaudeau, "Rule-Based Wood Knot Defect Image Classification," Proc. Masters Erasmus Mundus in Vision \& Robotics Meeting Day, pp. 87-91, 2010.

[8] P. Mohanaiah, et al., "Image Texture Feature Extraction Using GLCM Approach," International Journal of Scientific and Research Publications, vol/issue: 3(5), 2013.

[9] Prasetiyo, et al., "A Comparative Study of Feature Extraction Methods for Wood Texture Classification," Proc. of the 6th International Conference on Signal Image Technology and Internet Based Systems (SITIS), pp. 23-29, 2010.

[10] J. Y. Tou, et al., "Rotational invariant wood species recognition through wood species verification," Proc. of the 1 st Asian Conference on Intelligent Information and Database Systems, (DS '09), IEEE Xplore Press, Dong Hoi, pp. 115-120, 2009.

[11] Bremananth R., et al., "Wood Species Recognition System," International Journal of Computer, Electrical, Automation, Control and Information Engineering, vol/issue: 3(4), 2009.

[12] S. Mohan, et al., "An Intelligent Recognition System for Identification of Wood Species," Journal of Computer Science, vol/issue: 10(7), pp. 1231-1237, 2014.

[13] S. Mohan, et al., "Wood Species Identification System," International Journal of Engineering and Computer Science, vol/issue: 3(5), pp. 5996-6001, 2014

[14] M. Juneja and S. Shandu, "Performance Evaluation of Edge Detection Techniques for Images in Spatial Domain," International Journal of Computer Theory amd Engineering, vol/issue: 1(5), pp. 641-621, 2009.

[15] R. C. Gonzalez and R. E. Woods, "Digital Image Processing Third Edition," New York, Pearson Prentice Hall, 2008.

[16] R. Maini and H. Agwaral, "Study and Comaprison of Various Image Edge Detection Technique," International Journal of Image Processing (IJIIP), vol/issue: 3(1), 2009.

[17] M. Venkataramana, et al., "A Review of Recent Texture Classification: Methods," IOSR Journal of Computer Engineering (IOSR-JCE), vol/issue: 14(1), pp. 54-60, 2013.

[18] R. M. Haralick, et al., "Textual Features of Images Classification," IEEE Transaction on Systems, Man and Cybernatics, vol/issue: 3(6), pp. 610-621, 1973.

[19] A. Fahrurozi, et al., "Wood Texture Features Extraction by Using GLCM Combined With Various Edge Detection Methods", In The 2016 International Congress on Theoretical and Applied Mathematics, Physics \& Chemistry (The Science 2016), April 2016. 


\section{BIOGRAPHIES OF AUTHORS}
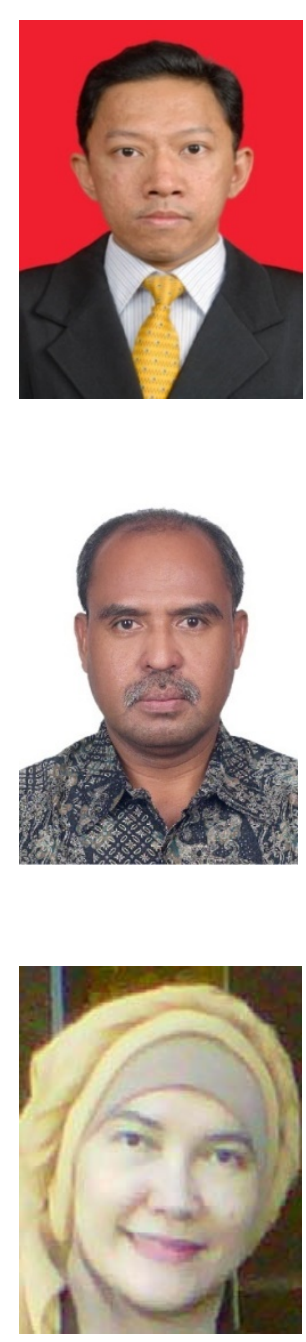

Dr. Ernastuti - has received B.S. degree in Mathematics from University of Indonesia in December 1985, and the M.S. in Computer Science from University of Indonesia, in July 1994, and $\mathrm{PhD}$ degrees in Computer Science from Gunadarma University, Indonesia, in April 2008. She is currently an associate professor in the faculty of computer science and Information Engineering, Gunadarma University. Her current research interests include graph theory and combinatorial optimization, graph-theoretic interconnection networks, parallel and distributed computing, and design and analysis of algorithms. Email: ernas_tuti@yahoo.co.id

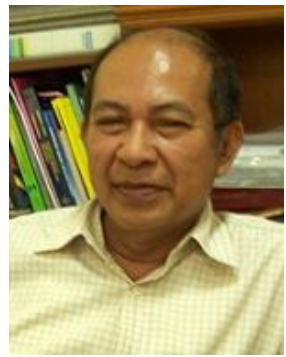

Djati Kerami - received the Doctor degree in informatics from Institute National Polytechnique de Toulouse, France, in 1985. He is working at Mathematics Department University of Indonesia as a professor. His current research interests include mathematical modeling in bioinformatics and

image processing algorithms. Email: djatikr@sci.ui.ac.id Makement Jakarta (STMIK Jakarta STI\&K). He received the B.S. degree from University of ndonesia, in 1989, the M.S. degree from Institute National des Sciences Appliquées de Lyon (InSA de Lyon) - French, in 1992, and the Ph.D. degree from University of Burgundy - French, Burgundy. In 1997, he became a Lecturer in Computer Science Department, Gunadarma University - Indonesia. From 2002 to 2007, he was a researcher at Academic Research Consortium on digital imaging, video, audio and multimedia (CoRIMedia), Canada. His research image processing: image compression, color imaging, image database and searching, medical image analysis, implementing of image processing algorithms on FPGA for real time image analysis. Email: sarif@staff.gunadarma.ac.id 